\title{
PGK1 is a Potential Survival Biomarker and Invasion Promoter by Regulating the HIF-1 $\alpha$-Mediated Epithelial-Mesenchymal Transition Process in Breast Cancer
}

\author{
Deyuan Fu Chunlan He ${ }^{a} \quad$ Jinli Wei ${ }^{a} \quad$ Zhengquan Zhang ${ }^{a} \quad$ Yulin Luo \\ Haosheng Tan ${ }^{\mathrm{a}}$ Chuanli Ren ${ }^{\mathrm{b}}$ \\ ${ }^{a}$ Department of Thyroid and Breast Surgery, Northern Jiangsu People's Hospital and Clinical Medical

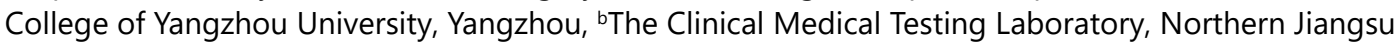 \\ People's Hospital and Clinical Medical College of Yangzhou University, Yangzhou, China
}

\section{Key Words}

Breast cancer $\cdot$ PGK1 $•$ HIF-1 $\alpha \cdot$ Epithelial-mesenchymal transition

\begin{abstract}
Background/Aims: Glycolysis, a multi-step enzymatic reaction, is considered to be the root of cancer development and progression. The aim of this study is to figure out which glycolysis enzyme participates in the progression of breast cancer and its possible mechanisms. Materials: We firstly screened out PGK1 by performing an RT-PCR array of glycolysis-related genes in three paired breast cancer samples, and further investigated PGK1 using TCGA and our own database. The effect and mechanism of PGK1 on cell invasion was further explored both in vitro and using patient samples. Results: PGK1 was most upregulated in T3N0 with distant metastases compared to those with no metastases. In the TCGA database, high PGK1 expression predicted poor overall survival (OS) in breast cancer and some other cancers $(P<0.001)$. In the validation cohort, high PGK1 expression was significantly correlated with larger tumor size $(P=0.011)$ and advanced TNM stage $(P=0.033)$, and PGK1 expression was an independent prognostic factor for OS and disease free survival (DFS) in both univariate and multivariate regression analyses $(P<0.05)$. Functional studies indicated that knockdown of PGK1 expression significantly inhibited invasion and reversed the epithelial-mesenchymal transition process in breast cancer cells $(P<0.05)$. Mechanistically, PGK1 increased HRE luciferase activity in a dose-dependent manner, while silencing PGK1 expression decreased HRE activity. Conclusion: High PGK1 expression was associated with poor prognosis in breast cancer, because PGK1 and HIF-1 $\alpha$ formed a positive feed-forward loop and thus stimulated breast cancer progression and metastases. Based on these results, PGK1 may serve as a promising biomarker and target therapy for breast cancer.

D. Fu and C. He contributed equally to this work.

\begin{tabular}{ll}
\hline Deyuan Fu, PhD, MD. & Department of Thyroid and Breast Surgery, Northern Jiangsu People's Hospital and Clinical \\
& Medical College of Yangzhou University, No. 98 Nantong West Road, Yangzhou 225001 (China) \\
& Tel. 86-0514-87373010, E-Mail fdy1003@163.com
\end{tabular}
\end{abstract}




\section{Cellular Physiology Cell Physiol Biochem 2018;51:2434-2444 \begin{tabular}{ll|l} 
and Biochemistry Published online: 8 December 2018 & $\begin{array}{l}\text { (c) } 2018 \text { The Author(s). Published by S. Karger AG, Basel } \\
\text { www.karger.com/cpb }\end{array}$ \\
\hline
\end{tabular} \\ Fu et al.: PGK1 in Breast Cancer}

\section{Introduction}

Cancer cells exhibit a series of common features, including uncontrolled proliferation, resistance to programmed cell death, and enhanced metastasis ability $[1,2]$. Of these, alteration of metabolic pathways is regarded as a driving force of tumor initiation and progression [2, 3]. Tumor cells always have a hypoxic microenvironment, and to overcome this harsh environment, cancer cells develop adaptive responses to hypoxia by activating hypoxia-inducible genes [4-6]. Hypoxia is a major characteristic of cancer, and in hypoxic conditions, metabolism in cancer cells is reprogrammed from oxidative phosphorylation to glycolysis [7, 8]. A feature of this phenomenon, called the Warburg effect or aerobic glycolysis, is increased lactate production even at normal oxygen concentrations. Many glucose metabolic enzymes are unregulated or downregulated in cancer tissues, and have been validated as multifaceted enzymes that are involved in various processes in cancer metastases and progression [9-12].

Phosphoglycerate kinase 1 (PGK1), a rate-limiting enzyme in the glycolysis process, catalyzes the conversion of 1, 3-diphosphoglycerate to 3-phosphoglycerate and generates a molecule of ATP [3]. PGK1 and pyruvate kinase M2 (PKM2) are the only enzymes that control production of ATP during aerobic glycolysis in cancer cells [1,3]. In addition to the important role of PGK1 in glycolysis, there are reports of its role in cancer. High PGK1 expression predicts poor survival in endometrial cancer [13] and gastric cancer [14]. However, Lu et al. demonstrated that low expression of PGK1 is associated with progression and correlated with poor prognosis in patients with gallbladder cancer [15].

Considering the conflicting results of PGK1 in different cancers and that the clinical significance of PGK1 in breast cancer has not been fully investigated, we focused on PGK1 in breast cancer progression and metastases. We found that high PGK1 expression predicted shorter survival in breast cancer and other cancers. An in vitro study found that silencing PGK1 impaired cell migration and the reverse epithelial-mesenchymal transition (EMT) process. Mechanistically, PGK1 and hypoxia-inducible factor 1-alpha (HIF1 $\alpha$ ) formed a positive loop that stimulated breast cancer progression.

\section{Materials and Methods}

\section{Patient samples}

Patients in TCGA. The TCGA gene expression database was used to study PGK1 in various cancers. TCGA is a project to catalogue gene expression or mutations responsible for cancer using bioinformatics methods and genome sequencing. To explore TCGA datasets, we used cBioPortal (http://www.cbioportal. org/index.do) to find pathways of interest in one or more cancer types [16-18]. Sequencing of the dataset was based on the Illumina HiSeq platform [19]. Inclusion criteria were as follows: patients diagnosed by pathological examination, with no pretreatment, with fully characterized tumors and intact overall survival (OS) information. Expression data of each PGK1 family were standardized, and the standardization between different samples was performed using the Limma package in R software [20].

Patient samples for primary screening. To find the glycolytic enzymes that may be involved in breast cancer progression, six breast primary tumor tissues were used in a PCR array for glycolytic enzymes. All primary tumors were pathologically diagnosed with T3N0 and were categorized as Luminal A. Three patients diagnosed with distant metastases were regarded as having high metastasis abilities, while three patients with no distant metastases were used as controls. Primary tumors were immediately put in RNA later and stored at $-20^{\circ} \mathrm{C}$ for further study.

Patients in the validation cohort. The patient samples used for external validation have been described previously [21]. All underwent intentionally curative surgical resection and received standard adjuvant therapy and follow up according to the National Comprehensive Cancer Network (NCCN) guidelines for breast cancer. All patients were restaged according to the $8^{\text {th }}$ edition of the TNM-UICC/AJCC classification. 


\section{Cellular Physiology Cell Physiol Biochem 2018;51:2434-2444 \begin{tabular}{l|l|l} 
DOl: 10.1159/000495900 & $\begin{array}{l}\text { O 2018 The Author(s). Published by S. Karger AG, Basel } \\
\text { www.karger.com/cpb }\end{array}$ \\
\hline
\end{tabular} \\ Fu et al.: PGK1 in Breast Cancer}

The OS was defined as the time from treatment to death from any cause, and the DFS was defined as the time from treatment to the first recurrence or death. Patients who were alive at last follow-up were censored for analysis.

\section{Cell culture}

Human breast cancer cell lines (MDA-MB-231 and MCF-7) were originally obtained from the American Type Culture Collection (ATCC; Rockville, MD, USA) and were cultured in Dulbecco's modified Eagle's medium (DMEM; Gibco-Invitrogen, Carlsbad, CA, USA) supplemented with 10\% fetal bovine serum (GibcoInvitrogen), $100 \mathrm{U} / \mathrm{ml}$ penicillin, and $100 \mu \mathrm{g} / \mathrm{ml}$ streptomycin. Cells were incubated at $37^{\circ} \mathrm{C}$ in a humidified incubator containing $5 \% \mathrm{CO}_{2}$.

\section{Lentivirus production and stable cell line selection}

To generate shRNA expression constructs against PGK1, a pLKO.1 TRC cloning vector (Plasmid10878, Addgene, Cambridge, MA, USA) was used. The 21 bp shRNA target against PGK1 was 5'GCAAGGATGTTCTGTTCTTGA -3'. pLK0.1-scramble shRNA (Plasmid1864, Addgene) with limited homology with any known sequences in humans was used as a negative control. Lentiviral particles were produced by co-transfection of pLK0.1-shPGK1 constructs with psPAX2 and pMD2.G into HEK-293T cells at a ratio of 4:3:1. Cell lines were obtained by infection of MDA-MB-231 and MCF-7 cells with lentiviral particles followed by puromycin selection.

Total RNA extraction and real-time polymerase chain reaction (real-time PCR)

Total RNA was extracted from breast cancer tissues and cells with the SV Total RNA Isolation system (Promega, Madison, WI, USA) according to the manufacturer's protocol. A PrimeScript ${ }^{\mathrm{TM}}$ RT Master Mix (Perfect Real Time) kit (RR036A, Takara) was used to synthesize first-strand cDNA from total RNA. mRNA expression was determined by real-time PCR using SYBR Green in an ABI 7900HT (Applied Biosystems, USA).

Sequences of PCR primer pairs were: human $\beta$-actin, forward 5' - CTACGTCGCCCTGGACTTCGAGC - 3 ' and reverse 5'- GATGGAGCCGCCGATCCACACGG -3'; and human PGK1, forward 5'-ATGCTGAGGCTGTCACTCGG-3' and reverse 5'-CACAGCAAGTGGCAGTGTCTCC-3'. Relative gene expression values were determined using the $2{ }^{-}{ }^{\Delta} \mathrm{CT}$ method as previously described [21]. The human $\beta$-actin gene was used as an endogenous control for sample normalization. Results were presented as fold increases relative to the expression of human $\beta$-actin.

\section{Western blot analysis}

Adherent cells were rinsed with deionized water and lysed with lysis buffer (RIPA Lysis Buffer, Thermo Scientific Pierce Protein Biology). Western blots were carried out as previously described in detail [21]. The membrane was either incubated overnight at $4^{\circ} \mathrm{C}$ in Tween-TBS-milk 5\% with primary antibodies PGK1 (1:1000; Abcam, Cambridge, UK), anti-E-cadherin antibody (ab40772, Abcam, 1:1000), anti-N-cadherin antibody (ab18203, Abcam, 1:1000), anti-vimentin (ab92547, Abcam, 1:1000), or mouse anti-human $\beta$-actin monoclonal antibody (ab133626, Abcam, 1:1000). After three washes with Tween-TBS and one with TBS, immunoreactivity was detected using enhanced chemiluminescence (Pierce, Thermo Scientific, USA).

\section{Immunohistochemical staining (IHC)}

Immunohistochemistry for PGK1 was performed as previously described [21]. Briefly, paraffin sections were baked for $60 \mathrm{~min}$ at $70^{\circ} \mathrm{C}$, de-paraffinized in xylene, rehydrated in an ethanol gradient $(75 \%, 95 \%$, $100 \%$ ), and then the sections were placed in $3 \% \mathrm{H}_{2} \mathrm{O}_{2}$ to neutralize endogenous peroxidase for $30 \mathrm{~min}$. Antigen retrieval was processed with citrate buffer $(\mathrm{pH}=6.0)$ in a pressure cooker. After antigen retrieval, the sections were incubated with PGK1 primary antibody (1:200; Abcam, Cambridge, UK) and secondary antibody. The sections were then stained with $\mathrm{DAB}$ (3, 3-diaminobenzidine) terminated in PBS, and then counterstained with hematoxylin. Based on the staining intensity of PGK1 in each case, the standards for scoring were as follows: 0 , negative; 1 , weak; 2 , moderate; and 3, strong. Zero and 1 were regarded as low expression and 2 and 3 were regarded as high expression. Two observers scored staining intensity independently. 


\section{Cellular Physiology Cell Physiol Biochem 2018;51:2434-2444 \begin{tabular}{ll|l} 
and Biochemistry & $\begin{array}{l}\text { DOI: 10.1159/000495900 } \\
\text { Published online: 8 December } 2018\end{array}$ & $\begin{array}{l}\text { ㄷ } 2018 \text { The Author(s). Published by S. Karger AG, Basel } \\
\text { www.karger.com/cpb }\end{array}$ \\
\hline
\end{tabular} \\ Fu et al.: PGK1 in Breast Cancer}

CCK8 assay

Cells were plated in 96-well plates at a density of $2 \times 10^{3}$ cells/well and cultured for 24, 48, 72, or $96 \mathrm{~h}$. Ten $\mu \mathrm{L}$ of CCK8 solution (Dojindo Laboratories, Kumamoto, Japan) was then added to each well, plates were incubated for $2 \mathrm{~h}$ at $37^{\circ} \mathrm{C}$ and read at $450 \mathrm{~nm}$. All experiments were performed three times.

Colony formation assay

Cells were seeded in six-well plates at a density of 200 per well and cultured at $37^{\circ} \mathrm{C}$ for 14 days. Cells were washed twice with PBS, fixed with $4 \%$ paraformaldehyde, stained with Giemsa for 10 minutes, and washed three times with double distilled $\mathrm{H}_{2} \mathrm{O}$. Colonies containing more than 50 cells were counted using light microscopy. Colony-forming efficiency (CFE \%) was defined as the ratio of the number of colonies formed in culture to the number of cells inoculated.

In vitro cell invasion assays

Invasion assays were conducted in a 24-well Transwell chamber (Costar, Cambridge, MA) with uncoated membranes or membranes coated with Matrigel (BD Biosciences, San Jose, CA, USA). Cells $\left(4 \times 10^{4}\right.$ per well) were resuspended in serum-free medium and seeded into the upper chamber. Culture medium containing $10 \%$ fetal bovine serum was added to the lower chamber as the chemoattractant. The cells were incubated in a humidified incubator at $37^{\circ} \mathrm{C}$ for 48 hours. Non-invading cells in the upper chambers were removed with cotton swabs. The cells attached to the lower surface were fixed and stained with crystal violet. The number of cells that attached to the lower surface was counted in five random fields under a microscope $(\times 200)$.

\section{Ethics statement}

This study was approved by the Northern Jiangsu People's Hospital Research Ethics Committee. The methods were carried out in accordance with the approved guidelines. Informed consent was obtained from each patient according to the committee's regulations.

\section{Statistical analysis}

The statistical associations between clinical parameters and IHC staining were tested using a $\chi^{2}$ test to compare the expected frequencies and the observed frequencies in two groups. The five-year OS and DFS were calculated with the Kaplan-Meier method to estimate the survival and hazard functions of censored data. The difference in survival between the groups at each observed event time was compared using the logrank test. Variables that seemed to be significantly associated with survival in univariate analysis were included in multivariate analysis, which was performed with the Cox proportional hazard model to relate survival to a collection of other risk factors. All in vitro experiments were performed independently with three replicates. A $P$ value of $<0.05$ was considered statistically significant. All statistical analyses were carried out using SPSS for Windows, version 21.0 (SPSS, Chicago, IL).

\section{Results}

PGK1 might promote tumor metastasis in breast cancer

Glycolysis is catalyzed by a series of glycolytic enzymes. To screen the candidate enzymes that may be involved in breast cancer progression and metastasis, an RT-PCR array was used to determine the mRNA levels of various glycolytic enzymes in three paired primary breast cancer tissues with or without distant metastases as mentioned above. The results indicated that the up-regulated expression of PGK1 and down-regulated expression of FBP1 were most significant in patients with distant metastases compared with those without distant metastases (Fig. 1A and 1B). As FBP1 has been thoroughly classified [10], we chose PGK1 for further study.

High PGK1 expression correlated with shorter OS in breast cancer and other tumors

As PGK1 may promote breast cancer metastases, we then asked whether PGK1 could predict survival in breast cancer. We first investigated PGK1 expression in the TCGA database 
Fig. 1. PGK1 is upregulated in patients with high invasive ability. (A) Heatmap of glycolytic genes in three paired breast cancer samples using RT-PCR with results normalized by Z-score. Each column represents a specimen denoted below and each row represents a gene denoted on the right. Red indicates genes that were upregulated and green indicates genes that were downregulated. P1-3: patients diagnosed with T3NOM0; P1'3': patients diagnosed with T3N0M1. (B) PGK1 was most upregulated, while FBP1 was significantly downregulated in T3N0M1 patients compared to those diagnosed with T3N0M1.
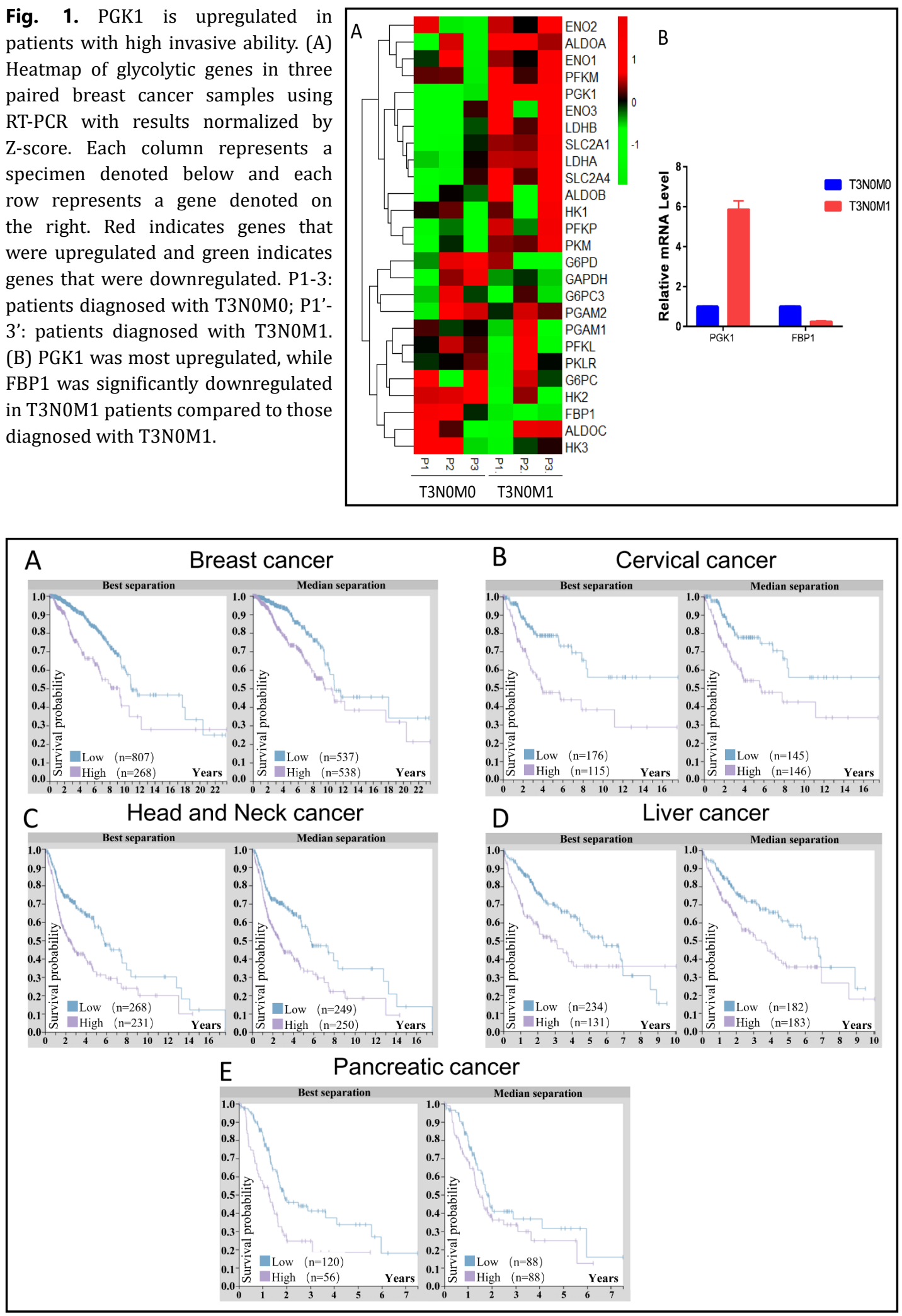

Fig. 2. PGK1 is a novel biomarker for survival in various cancers. High PGK1 implies poor survival in breast cancer (A), cervical cancer (B), head and neck cancer (C), liver cancer (D), and pancreatic cancer (E). Patients were divided into PGK1 high or low expression subgroups with optimal cutoff (left panel) or median value (right value). All $\mathrm{P}<0.001$. 
and found that high PGK1 expression predicts poor survival in breast cancer (HR: 1.984, 95\% CI 1.481-2.656, $P<0.001$ ). PGK1 mRNA expression levels had a nearly normal distribution, so we then divided the patients into high and low PGK1 expression subgroups based on the median level. The five year OS was $88.2 \%$ and $73.1 \%$ for patients in the high and low PGK1 expression groups, respectively $\left(\chi^{2}=8.751\right.$, $P=0.003$ ) (Fig. 2A). Furthermore, we found that high PGK1 expression also implied short OS in head and neck cancer, cervical cancer, liver cancer, and pancreatic cancer using the THPA database (Fig. 2B-2E).

\section{PGK1 was an independent prognostic factor} for survival in the validation cohort

A total of 185 patients were used for external validation of clinical significance of PGK1 in breast cancer. Patient characteristics are shown in Table 1. High PGK1 expression was found in 124 (67.0\%) patients. Representative figures are in Fig. 3A. Chi-square analysis demonstrated that high PGK1 expression was significantly correlated with larger tumor size $(P=0.011)$ and advanced TNM stage $(P=0.033)$ (Fig. 3B and 3C) (Table 1).

Table 1. Comparison of baseline clinical characteristics based on PGK1 expression level

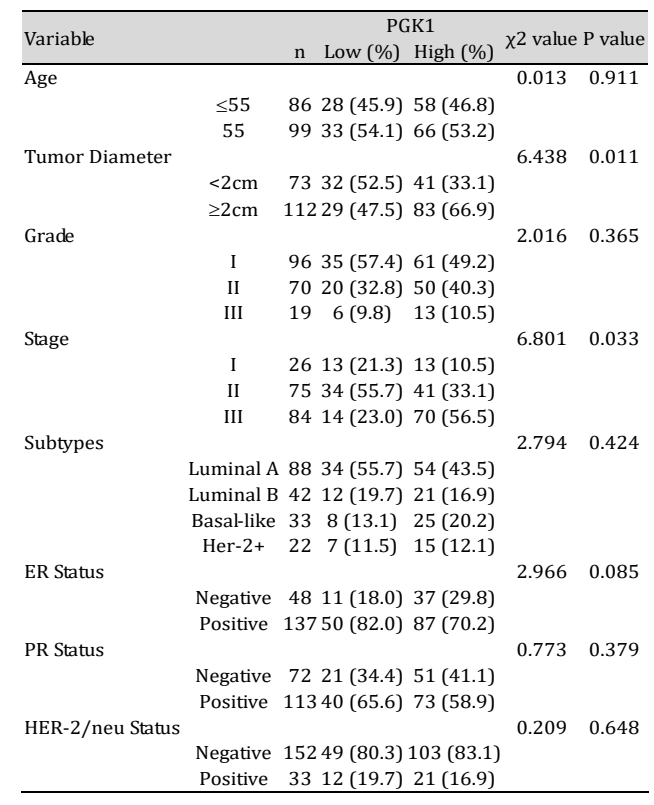

Fig. 3. High PGK1 expression predicts poor survival in validation cohorts. (A) Representative images of high (upper panel) and low (lower panel) PGK1 expression in breast cancer. The original figure was $100 \mathrm{X}$ in the left panel, amplified to $200 \mathrm{X}$ in the right panel. (B, C) High PGK1 expression was significantly correlated with larger tumor size (B) and advanced tumor stage (C). KaplanMeier analyses were performed to evaluate the correlation of PGK1 expression and patients' survival. Overexpression of PGK1 was correlated with worse OS (D) and DFS (E) $(\mathrm{P}<0.001)$.

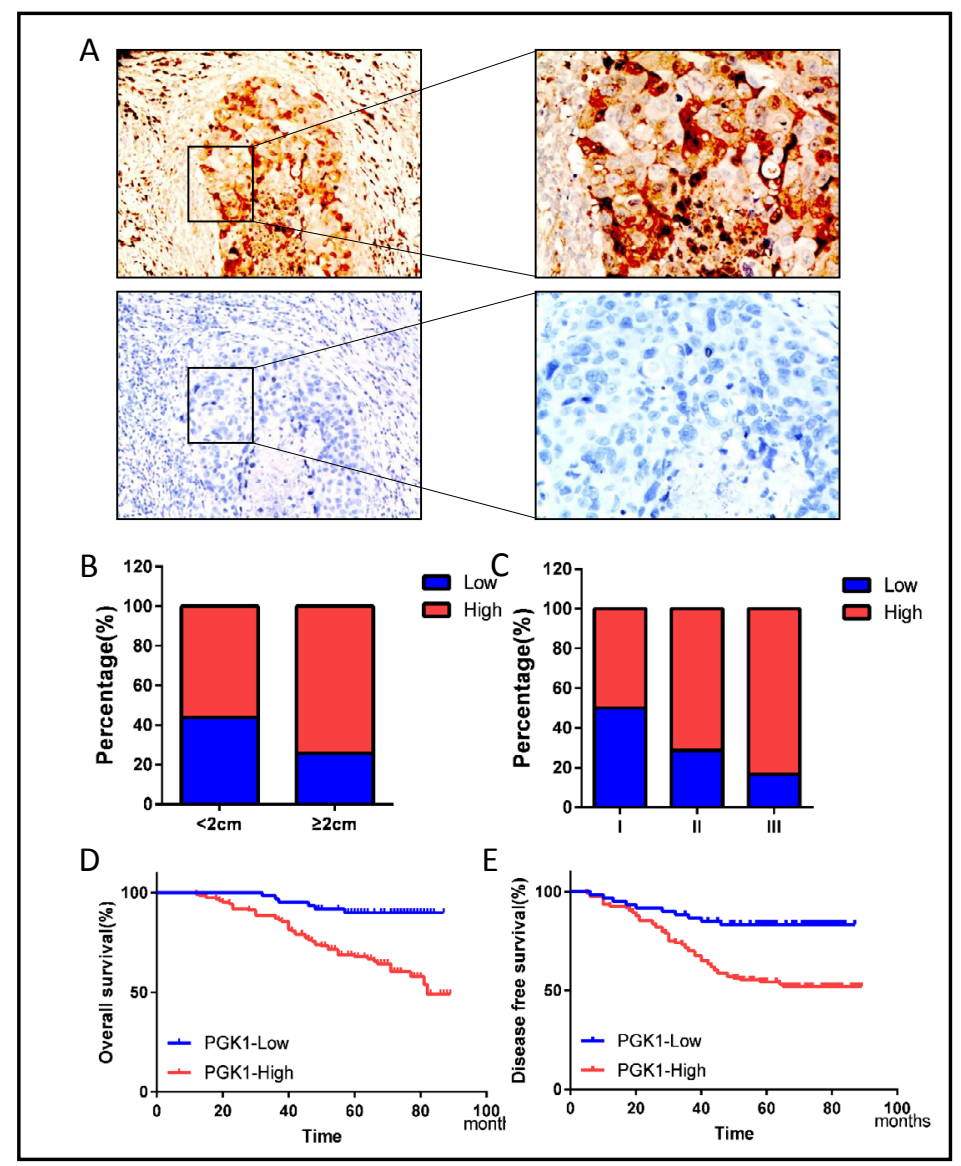


Five-year OS in the high PGK1 expression subgroup was $67.9 \%$ compared with $90.0 \%$ in the low expression subgroup. The log-rank analysis showed that the differences were significant $\left(\chi^{2}=14.694, P<0.001\right.$, Fig. 3D). Similarly, patients with low PGK1 expression levels showed better five-year DFS than those with high expression $\left(83.4 \%\right.$ vs. $54.5 \%, \chi^{2}=13.984$, $\mathrm{P}<0.001$ ) (Fig. 3E). Furthermore, multivariate Cox regression analysis showed that PGK1 expression was an independent factor for predicting both OS (HR: 3.155, 95\% CI: 1.316 -7.569, $\mathrm{P}=0.010$ ) (Table 2) and DFS (HR: 2.229, 95\% CI: $1.105-4.499, \mathrm{P}=0.025$ ) (Table $3)$. These data further confirmed that PGK1 is a potential prognostic marker in clinical practice.
Table 2. Univariate and multivariate survival analyses of PGK1 expression and overall survival for patients with breast cancer. NI: not included in multivariate survival analysis

\begin{tabular}{lcccc}
\hline \multirow{2}{*}{ Variable } & \multicolumn{2}{c}{ Univariate analysis } & \multicolumn{2}{c}{ Multivariate analysis } \\
& HR $(95 \% \mathrm{CI})$ & $\mathrm{P}$ & $\mathrm{HR}(95 \% \mathrm{CI})$ & $\mathrm{P}$ \\
\hline Age & $1.022(0.997-1.048)$ & 0.090 & & $\mathrm{NI}$ \\
Tumor Diameter & $3.748(1.829-7.681)$ & $<0.001$ & $2.763(1.337-5.710)$ & 0.006 \\
Grade & $1.955(1.346-2.840)$ & $<0.001$ & $1.777(1.192-2.649)$ & 0.005 \\
Stage & $2.991(1.790-4.999)$ & $<0.001$ & $1.637(1.123-2.749)$ & 0.018 \\
Subtypes & $1.206(0.952-1.528)$ & 0.121 & & $\mathrm{NI}$ \\
ER Status & $0.532(0.305-0.927)$ & 0.026 & $0.845(0.478-1.493)$ & 0.561 \\
PR Status & $1.003(0.578-1.740)$ & 0.992 & & $\mathrm{NI}$ \\
HER-2/neu Status & $1.055(0.530-2.102)$ & 0.879 & & $\mathrm{NI}$ \\
PGK1 & $4.528(1.934-10.598)$ & 0.001 & $3.155(1.316-7.569)$ & 0.010 \\
\hline
\end{tabular}

Table 3. Univariate and multivariate survival analyses of PGK1 expression and disease free survival for patients with breast cancer. NI: not included in multivariate survival analysis

\begin{tabular}{lcccc}
\hline \multirow{2}{*}{ Variable } & \multicolumn{2}{c}{ Univariate analysis } & \multicolumn{2}{c}{ Multivariate analysis } \\
& HR $(95 \% \mathrm{CI})$ & $\mathrm{P}$ & HR (95\%CI) & $\mathrm{P}$ \\
\hline Age & $1.027(1.004-1.050)$ & 0.020 & $1.699(1.023-2.820)$ & 0.040 \\
Tumor Diameter & $2.722(1.534-4.832)$ & 0.001 & $2.055(1.148-3.677)$ & 0.015 \\
Grade & $1.899(1.359-2.654)$ & $<0.001$ & $1.572(1.093-2.259)$ & 0.015 \\
Stage & $2.705(1.762-4.152)$ & $<0.001$ & $1.689(1.082-2.636)$ & 0.021 \\
Subtypes & $1.279(1.014-1.573)$ & 0.019 & $1.139(0.912-1.432)$ & 0.250 \\
ER Status & $0.595(0.360-0.985)$ & 0.043 & $0.918(0.534-1.578)$ & 0.756 \\
PR Status & $0.856(0.530-1.385)$ & 0.527 & & $\mathrm{NI}$ \\
HER-2/neu Status & $0.808(0.413-1.580)$ & 0.533 & & $\mathrm{NI}$ \\
PGK1 & $3.323(1.697-6.504)$ & $<0.001$ & $2.229(1.105-4.499)$ & 0.025 \\
\hline
\end{tabular}

Fig. 4. Silencing PGK1 expression inhibits invasion and reverses the EMT process in breast cancer. PGK1 expression was silenced by shRNA in breast cancer cells, and the knockdown efficacy was determined by Western blotting (A) and RT-PCR (B). Knockdown PGK1 expression significantly inhibited cell invasive abilities (C). Western blot (D) and RT-PCR (E) analyses indicated that knockdown of PGK1 expression significantly increased the expression level of E-cadherin and decreased the levels of vimentin and $\mathrm{N}$-cadherin. ${ }^{*} \mathrm{P}<0.05$.

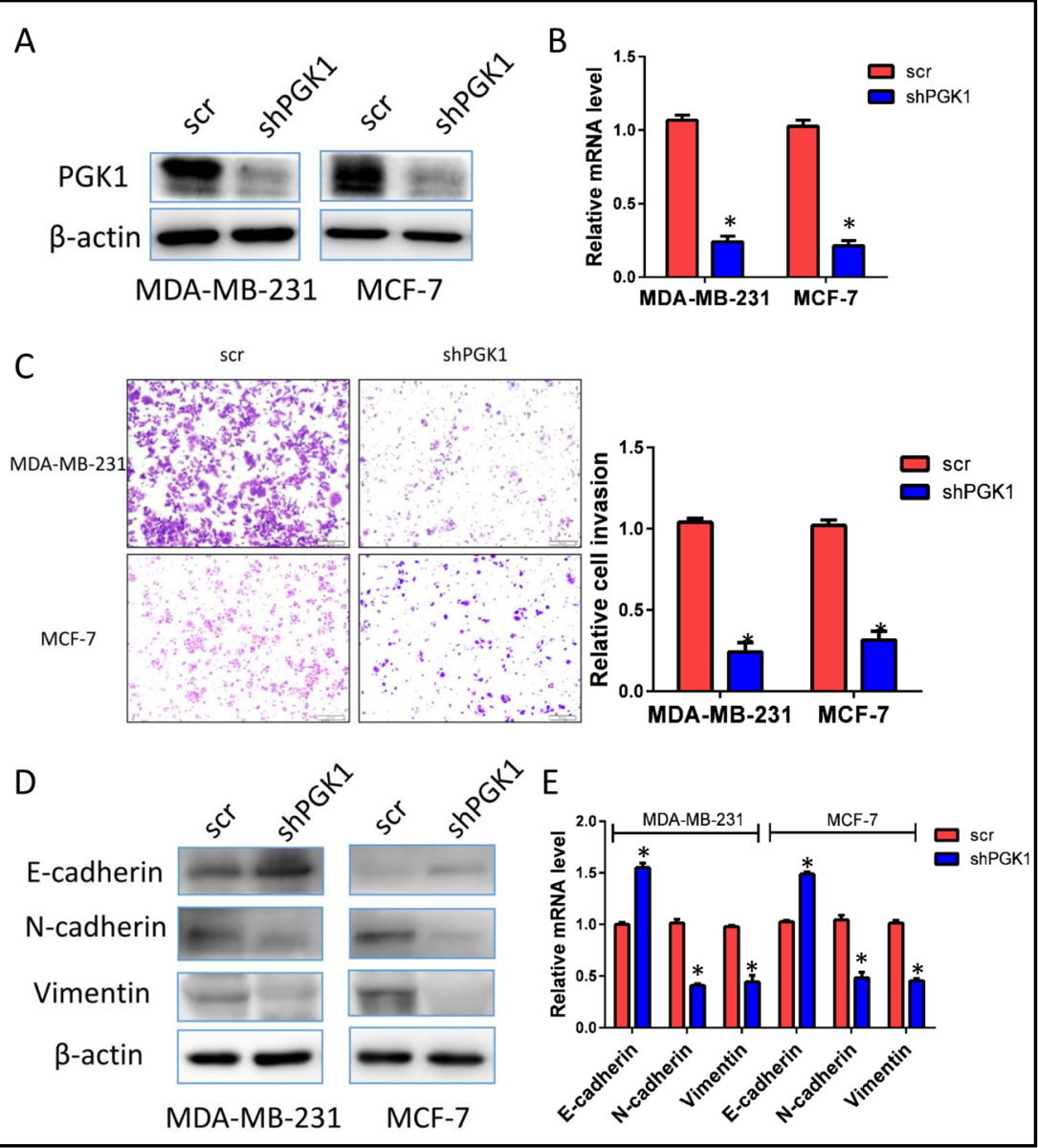


Silencing PGK1
expression impaired
breast cancer cell
invasion and the reverse
EMT process
To assess the role of PGK1 in regulating breast cell invasion, PGK1 expression was silenced by lentivirusmediated transfection. Western blot and RT-PCR analysis demonstrated that PGK1 expression was significantly decreased at both the transcription level and the protein level in MDA-MB-231 and MCF7 cells (Fig. 4A and 4B). To assess the role of PGK1 in cell viability, transwell analysis was performed, and our results demonstrated that knockdown of PGK1 expression significantly attenuated cell invasion abilities (Fig. 4C). To determine whether it was EMT that mediated the effect of PGK1 on invasion, we examined the expression of several EMT markers in PGK1 knockdown cells. As expected, silencing PGK1 expression increased the expression level of E-cadherin and decreased the levels of vimentin and $\mathrm{N}$-cadherin (Fig. 4D). In the TCGA database, there was also a significant relationship among PGK1 and EMT-related markers (Fig. 4E).

\section{PGK1 and HIF-1 $\alpha$ formed a positive} feedback loop to promote metastases in breast cancer

PGK1 is a transcriptional target of HIF- $1 \alpha$, and glycolysis is necessary for maintaining HIF- $1 \alpha$ activity. So, we hypothesized that PGK1 can affect HIF- $1 \alpha$ activity in breast cancer. HIF- $1 \alpha$ acts by binding to HIF- $1 \alpha$ response element (HRE) upon hypoxia; we thereby utilized the HRE-luciferase reporter to examine whether PGK1 affects HRE luciferase activities. As expected, PGK1 increased HRE luciferase activity in a dose-dependent manner (Fig. 5A), while silencing PGK1 expression decreased HRE activity (Fig. 5B). Moreover, we observed a close relationship between PGK1 and HIF-1 $\alpha$ expression in 185 breast cancer samples (Fig. 5C and Table 4). In addition, the PGK1 mRNA expression level was significantly correlated with the HIF-1 $\alpha$ mRNA level in the TCGA database (Fig. 5D), implying that HIF-1 $\alpha$ and PGK1 could form a positive feedback loop to promote metastases in breast cancer.

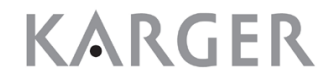




\section{Cellular Physiology Cell Physiol Biochem 2018;51:2434-2444 \begin{tabular}{ll|l} 
and Biochemistry & $\begin{array}{l}\text { DOl: 10.1159/000495900 } \\
\text { Published online: 8 December } 2018\end{array}$ & $\begin{array}{l}\text { O } 2018 \text { The Author(s). Published by S. Karger AG, Basel } \\
\text { www.karger.com/cpb }\end{array}$ \\
\cline { 2 - 3 }
\end{tabular} \\ Fu et al.: PGK1 in Breast Cancer}

\section{Discussion}

Glycolytic enzymes have been widely studied as potential therapeutic targets because cancer-specific metabolism depends more on the glycolytic pathway than on aerobic respiration, which has been regarded as one of the ten hallmarks of cancer [2]. In the present study, we first screened out PGK1 by performing RT-PCR arrays of glycolysis-related genes in three different samples with paired metastasis abilities in primary breast cancer tissues. We then explored PGK1 expression using the TCGA database and found that high PGK1 implied poor survival in breast cancer and other cancers, suggesting that unregulated glycolytic enzymes, especially PGK1, is a common phenomenon in cancer.

The TCGA database lacks some important information, such as the quality of surgery and adjuvant therapy strategies, and thus only the transcriptional expression level of PGK1 is available in the TCGA database. We further validated the clinical significance of PGK1 expression with our own database using IHC. Our results demonstrated that high PGK1 expression was significantly correlated with larger tumor size and advanced tumor stage, which suggested that PGK1 may play a critical role in breast cancer progression and metastases. Importantly, our study indicated that PGK1 expression was an independent prognosis biomarker in breast cancer after radical resection.

A functional study indicated that silencing PGK1 expression significantly impaired the ability to invade breast cancer cells. EMT is considered critical for invasive and metastatic progression in cancer. The process of EMT is associated with the downregulation of epithelial markers and aberrant upregulation of mesenchymal markers. As anticipated, knockdown of PGK1 expression significantly upregulated epithelial marker E-cadherin and downregulated mesenchymal markers $\mathrm{N}$-cadherin and vimentin. Some studies have demonstrated that glycolytic enzymes are involved in the EMT process, including FBP1 [10, 11, 22], PKM2 [23], and LDHA. Glycolytic enzymes are also regulated by classic EMT regulators [11, 24-26], so, it is not surprising that PGK1 can regulate the EMT process in breast cancer.

HIF- $1 \alpha$ is central regulator for cancer cell survival and metastases in solid tumors in part by inducing glycolysis and the EMT process [27-29]. HIF-1 $\alpha$ upregulates a series of glycolytic genes during anaerobic glycolysis and has PGK1 as a downstream target. A previous study also suggested that glycolysis is necessary for maintaining HIF-1 $\alpha$ activity [30]. Here, we demonstrated that PGK1 increased HIF- $1 \alpha$ activity. PGK1 and HIF- $1 \alpha$ formed a positive feedforward loop that stimulates breast cancer progression and metastases. Thus, PGK1 may serve as a promising biomarker and target therapy for breast cancer.

A major strength of this study is that we first screened out PGK1 using a PCR array from breast cancer samples with different invasion abilities and validated clinical significance of PGK1 from two independent populations. PGK1 is not only upregulated in breast cancer, but also in several other tumors. We also used an in vitro study and a mechanistic study to explore the underlying mechanism of how PGK1 promotes progression and metastases. However, there are some limitations in our study. First, no in vivo experiments using animals were performed to observe the effects of PGK1 on progression and metastases from mouse models. Thus, further in vivo animal studies are warranted to confirm our findings. Second, some glycolytic enzymes are involved in drug resistance and radiotherapy resistance, but whether PGK1 has a similar effect requires further study.

\section{Conclusion}

High PGK1 expression was associated with poor prognosis in breast cancer. PGK1 and HIF- $1 \alpha$ formed a positive feed-forward loop that stimulated breast cancer progression and metastases. Thus, PGK1 may serve as a promising biomarker and target therapy for breast cancer. 


\section{Cellular Physiology Cell Physiol Biochem 2018;51:2434-2444

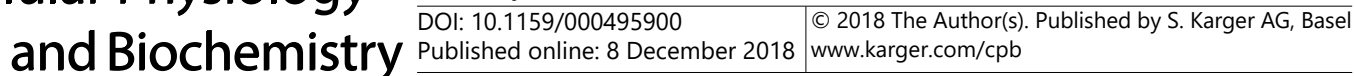 Fu et al.: PGK1 in Breast Cancer}

\section{Acknowledgements}

This study was partially supported by grants from the National Science Foundation of China (No. 81172508). The funders had no role in the study design, data collection and analysis, decision to publish, or preparation of the manuscript.

\section{Disclosure Statement}

The authors declare no conflicts of interest.

\section{References}

1 Hu H, Zhu W, Qin J, Chen M, Gong L, Li L, Liu X, Tao Y, Yin H, Zhou H, Zhou L, Ye D, Ye Q Gao D: Acetylation of PGK1 promotes liver cancer cell proliferation and tumorigenesis. Hepatology 2017;65:515-528.

2 Hanahan D, Weinberg RA: Hallmarks of cancer: the next generation. Cell 2011;144:646-674.

3 Warburg 0: On the origin of cancer cells. Science 1956;123:309-314.

4 Kawai K, Uemura M, Munakata K, Takahashi H, Haraguchi N, Nishimura J, Hata T, Matsuda C, Ikenaga M, Murata K, Mizushima T, Yamamoto H, Doki Y, Mori M: Fructose-bisphosphate aldolase A is a key regulator of hypoxic adaptation in colorectal cancer cells and involved in treatment resistance and poor prognosis. Int J Oncol 2017;50:525-534.

5 Nagaraju GP, Bramhachari PV, Raghu G, El-Rayes BF: Hypoxia inducible factor-1alpha: Its role in colorectal carcinogenesis and metastasis. Cancer Lett 2015;366:11-18.

6 Schofield CJ, Ratcliffe PJ: Oxygen sensing by HIF hydroxylases. Nat Rev Mol Cell Biol 2004;5:343-354.

7 Denko NC: Hypoxia, HIF1 and glucose metabolism in the solid tumour. Nat Rev Cancer 2008;8:705-713.

-8 DeBerardinis RJ, Lum JJ, Hatzivassiliou G, Thompson CB: The biology of cancer: metabolic reprogramming fuels cell growth and proliferation. Cell Metab 2008;7:11-20.

-9 Li Q Li Y, Xu J, Wang S, Xu Y, Li X, Cai S: Aldolase B Overexpression is Associated with Poor Prognosis and Promotes Tumor Progression by Epithelial-Mesenchymal Transition in Colorectal Adenocarcinoma. Cell Physiol Biochem 2017;42:397-406.

10 Dong C, Yuan T, Wu Y, Wang Y, Fan TW, Miriyala S, Lin Y, Yao J, Shi J, Kang T, Lorkiewicz P, St Clair D, Hung MC, Evers BM, Zhou BP: Loss of FBP1 by Snail-mediated repression provides metabolic advantages in basal-like breast cancer. Cancer Cell 2013;23:316-331.

11 Yu J, Li J, Chen Y, Cao W, Lu Y, Yang J, Xing E: Snail Enhances Glycolysis in the Epithelial-Mesenchymal Transition Process by Targeting FBP1 in Gastric Cancer. Cell Physiol Biochem 2017;43:31-38.

12 Alderton GK: Tumorigenesis: FBP1 is suppressed in kidney tumours. Nat Rev Cancer 2014;14:575.

13 Guo S, Xiao Y, Li D, Jiang Q, Zhu L, Lin D, Jiang H, Chen W, Wang L, Liu C, Fang W, Lin L: PGK1 and GRP78 overexpression correlates with clinical significance and poor prognosis in Chinese endometrial cancer patients. Oncotarget 2018;9:680-690.

14 Zieker D, Konigsrainer I, Tritschler I, Loffler M, Beckert S, Traub F, Nieselt K, Buhler S, Weller M, Gaedcke J, Taichman RS, Northoff H, Brucher BL, Konigsrainer A: Phosphoglycerate kinase 1 a promoting enzyme for peritoneal dissemination in gastric cancer. Int J Cancer 2010;126:1513-1520.

15 Lu W, Gao J, Yang J, Cao Y, Jiang L, Li M, Zhang Y, Zhou J, Liu Y: Down-Regulated Phosphoglycerate Kinase 1 Expression Is Associated With Poor Prognosis in Patients With Gallbladder Cancer. Medicine (Baltimore) 2015;94:e2244.

16 Cerami E, Gao J, Dogrusoz U, Gross BE, Sumer SO, Aksoy BA, Jacobsen A, Byrne CJ, Heuer ML, Larsson E, Antipin Y, Reva B, Goldberg AP, Sander C, Schultz N: The cBio cancer genomics portal: an open platform for exploring multidimensional cancer genomics data. Cancer Discov 2012;2:401-404.

17 Gao J, Aksoy BA, Dogrusoz U, Dresdner G, Gross B, Sumer SO, Sun Y, Jacobsen A, Sinha R, Larsson E, Cerami E, Sander C, Schultz N: Integrative analysis of complex cancer genomics and clinical profiles using the cBioPortal. Sci Signal 2013;6:pl1.

18 Jiang Z, Yu T, Fan Z, Yang H, Lin X: Kruppel-Like Factor 7 is a Marker of Aggressive Gastric Cancer and Poor Prognosis. Cell Physiol Biochem 2017;43:1090-1099. 


\section{Cellular Physiology Cell Physiol Biochem 2018;51:2434-2444

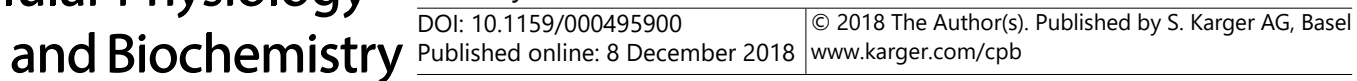 \\ Fu et al.: PGK1 in Breast Cancer}

19 Quail MA, Smith M, Coupland P, Otto TD, Harris SR, Connor TR, Bertoni A, Swerdlow HP, Gu Y: A tale of three next generation sequencing platforms: comparison of Ion Torrent, Pacific Biosciences and Illumina MiSeq sequencers. BMC Genomics 2012;13:341.

20 Autio R, Kilpinen S, Saarela M, Kallioniemi O, Hautaniemi S, Astola J: Comparison of Affymetrix data normalization methods using 6, 926 experiments across five array generations. BMC Bioinformatics 2009;10 Suppl 1:S24.

-21 Fu D, Li J, Wei J, Zhang Z, Luo Y, Tan H, Ren C: HMGB2 is associated with malignancy and regulates Warburg effect by targeting LDHB and FBP1 in breast cancer. Cell Commun Signal 2018;16:8.

-22 Li J, Wang Y, Li QG, Xue JJ, Wang Z, Yuan X, Tong JD, Xu LC: Downregulation of FBP1 Promotes Tumor Metastasis and Indicates Poor Prognosis in Gastric Cancer via Regulating Epithelial-Mesenchymal Transition. PLoS One 2016;11:e0167857.

23 Hamabe A, Konno M, Tanuma N, Shima H, Tsunekuni K, Kawamoto K, Nishida N, Koseki J, Mimori K, Gotoh $\mathrm{N}$, Yamamoto H, Doki Y, Mori M, Ishii H: Role of pyruvate kinase M2 in transcriptional regulation leading to epithelial-mesenchymal transition. Proc Natl Acad Sci U S A 2014;111:15526-15531.

-24 Kim NH, Cha YH, Lee J, Lee SH, Yang JH, Yun JS, Cho ES, Zhang X, Nam M, Kim N, Yuk YS, Cha SY, Lee Y, Ryu JK, Park S, Cheong JH, Kang SW, Kim SY, Hwang GS, Yook JI, Kim HS: Snail reprograms glucose metabolism by repressing phosphofructokinase PFKP allowing cancer cell survival under metabolic stress. Nat Commun 2017;8:14374.

25 Li Q, Qin Y, Wei P, Lian P, Li Y, Xu Y, Li X, Li D, Cai S: Gas1 Inhibits Metastatic and Metabolic Phenotypes in Colorectal Carcinoma. Mol Cancer Res 2016;14:830-840.

-26 Cui J, Shi M, Xie D, Wei D, Jia Z, Zheng S, Gao Y, Huang S, Xie K: FOXM1 promotes the warburg effect and pancreatic cancer progression via transactivation of LDHA expression. Clin Cancer Res 2014;20:25952606.

-27 Zhang W, Shi X, Peng Y, Wu M, Zhang P, Xie R, Wu Y, Yan Q, Liu S, Wang J: HIF-1alpha Promotes EpithelialMesenchymal Transition and Metastasis through Direct Regulation of ZEB1 in Colorectal Cancer. PLoS One 2015;10:e0129603.

28 Zhang L, Huang G, Li X, Zhang Y, Jiang Y, Shen J, Liu J, Wang Q, Zhu J, Feng X, Dong J, Qian C: Hypoxia induces epithelial-mesenchymal transition via activation of SNAI1 by hypoxia-inducible factor -1alpha in hepatocellular carcinoma. BMC Cancer 2013;13:108.

29 Robey IF, Lien AD, Welsh SJ, Baggett BK, Gillies RJ: Hypoxia-inducible factor-1alpha and the glycolytic phenotype in tumors. Neoplasia 2005;7:324-330.

-30 Grandjean G, de Jong PR, James BP, Koh MY, Lemos R, Kingston J, Aleshin A, Bankston LA, Miller CP, Cho EJ, Edupuganti R, Devkota A, Stancu G, Liddington RC, Dalby KN, Powis G: Definition of a Novel Feed-Forward Mechanism for Glycolysis-HIF1alpha Signaling in Hypoxic Tumors Highlights Aldolase A as a Therapeutic Target. Cancer Res 2016;76:4259-4269. 